\title{
Long-Term Survival and Complete Response to Anastrozole in Breast Cancer Patient With Cutaneous Metastases Only
}

\author{
THEOPHIL SEDLOEV ${ }^{1}$, ALEXANDRINA VLAHOVA ${ }^{2}$, SLAVYANA USHEVA ${ }^{1}$, \\ MARIELA VASILEVA-SLAVEVA ${ }^{3}$, TSVETELINA SPIRIDONOVA ${ }^{1}$ and JORDAN SPIRDONOV ${ }^{4}$ \\ ${ }^{1}$ Department of Surgery, University Hospital "Tzaritza Joanna - ISUL”, \\ Medical University of Sofia, Sofia, Bulgaria; \\ ${ }^{2}$ Department of Pathology, Medical University of Sofia, Sofia, Bulgaria; \\ ${ }^{3}$ Department of Surgery, University Hospital "Alexandrovska", Medical University of Sofia, Sofia, Bulgaria; \\ ${ }^{4}$ Department of Medical Imaging, University Hospital "Tzaritza Joanna - ISUL", \\ Medical University of Sofia, Sofia, Bulgaria
}

\begin{abstract}
Background/Aim: Metastatic breast cancer (MBC) represents a wide spectrum of heterogeneous conditions with different secondary spread sites, time to relapse and biology. $M B C$ is still considered an incurable disease despite the fact that survival rates have improved in the last years. Cutaneous metastases are a rare site for metastatic spread and indicate advanced disease. The aim of this study is to demonstrate the excellent therapeutic result following endocrine therapy only in MBC with just skin involvement. Case Report: We present a case of an 82-year-old woman with no family history of breast cancer $(B C)$, who was diagnosed with de novo metastatic estrogen/progesterone receptor-positive and HER2-negative invasive lobular BC. The only site of secondary spread was the skin. She was treated with just endocrine therapy for 116 months with which she achieved and maintained long-term complete clinical response (CR). Discussion: To our knowledge this is the only case of lobular BC with de novo metastatic manifestation as multiple skin metastases, which achieved $C R$ following the aromatase inhibitor treatment (anastrozole) with such impressive long-term overall survival.
\end{abstract}

Breast cancer (BC) is the most common malignancy in women worldwide. Most patients diagnosed with early $\mathrm{BC}$ have a longterm survival, but despite optimal treatment, nearly $30 \%$ relapse

This article is freely accessible online.

Correspondence to: Theophil Sedloev, Department of Surgery, University Hospital "Tzaritza Joanna - ISUL", Medical University of Sofia, Str. "Byalo More" 8, 1527 Sofia, Bulgaria. Tel: +359 888624327, e-mail: theosed@abv.bg

Key Words: Cutaneous metastatic breast cancer, endocrine therapy, complete response, anastrozole. or progress (1-3). The prognosis in the metastatic setting is more limited. Skin lesions are a rare site for metastatic spread and indicate advanced disease $(4,5)$. In about $0.8 \%$ of all cancers skin lesions may be the first clinical manifestation of malignancy (6). Of all solid tumors skin metastases (SM) are most frequently seen in BC and they most frequently occur on the anterior chest wall followed by the skin of the contralateral breast, scar sites, the arms, and the skin of the head and neck (7-9). Approximately $6 \%$ of the patients with $\mathrm{BC}$ are diagnosed with de novo distant metastases and in only $6 \%$ of them their cutaneous lesions are the only metastatic site (10). Cutaneous metastases as a single metastatic site predict a comparatively good prognosis for patients with hormone receptor positive tumors with a median survival of 55 months compared to 29.4 months for all de novo metastatic $\mathrm{BC}$ patients $(11,12)$.

\section{Case Presentation}

We present a case of an 82-year-old woman with no family history of BC, who was diagnosed in April 2007 with a tumor mass in her left breast. The lesion was localized in the central part, involving almost the entire breast. Involvement of the nipple-areolar complex and the skin was visible with a macroscopic view as in Paget's disease. There was a single enlarged lymph node, measuring $12 \mathrm{~mm}$ in the ipsilateral left axilla and no enlarged lymph nodes were present in the supraclavicular region. There were multiple nodular lesions on the skin of the same breast, the abdominal wall and the back (Figure 1).

The mammography showed a single 34-mm tumor lesion, involving the whole central part of the left breast. The initial staging was done using an abdominal ultrasound, chest X-ray and bone scintigraphy that did not show any visceral or bone metastases. The process was thus classified as de novo stage IV - cT4N1M1 (skin), according to AJCC Cancer Staging 
Manual, 6th Edition (13). The only abnormal laboratory findings were the elevated levels of tumor markers CA 15-3 $111.73 \mathrm{U} / \mathrm{ml}$ (reference ranges 0-37 U/ml) and CEA - 8.56 $\mathrm{ng} / \mathrm{ml}$ (reference ranges $0-5.5 \mathrm{ng} / \mathrm{ml}$ ). Listed co-morbidities were: i) asymptomatic mild lung emphysema and ii) pneumofibrosis that were both untreated. The initial osteodensitometry showed mild osteopenia (T- score -, according to World Health Organization - WHO Criteria for Diagnosis of Osteoporosis) (14). Her Eastern Cooperative Oncology Group Performance Status (ECOGPS) was 1 and her body mass index (BMI) was 23. The patient did not take any medication until the diagnosis of $\mathrm{BC}$.

The histological analysis of the incisional biopsy of the tumor and the excisional one of a single skin lesion showed a classical moderately differentiated G2 invasive lobular carcinoma with skin infiltration and SM (Figure 2A and B). The immuno staining showed luminal A-like staining with strong homogeneous positivity for estrogen (ER) - 7/8 and progesterone (PR) - 6/8 (Figure 3A and B). Expression of HER2, p53 or E -cadherin was absent. The proliferative activity index marker, Ki-67, was at $16 \%$ and there was strong positive cytoplasmic expression of B-cell lymphoma 2 (bcl-2).

Immunostaining for ER, PgR, HER2 protein, p 53, bcl-2, and E-cadherin and Ki-67 (DakoCytomation, Denmark) was carried out on $4 \mu$ sections from the paraffin embedded tissue according to the manufacturer protocol. The following primary antibodies were used: the mAb to ER $\alpha$ (Clone SP1, at $1 / 200$ dilution), the mAb to PgR (Clone PgR 636 Dako, 1/50), the pAb to HER2 (Clone C - erb B 2 Oncoprotein $\mathrm{SP} 1$, at 1/300 dilution), the mAb to the Ki-67 antigen (Clone MIB1, DakoCytomation), the mAb p53 protein (Clone DO7, DakoCytomation), the mAb BCL-2 Oncoprotein (Clone 124, DakoCytomation), the mAb to E-cadherin (Clone NCH-38, DakoCytomation).

The multidisciplinary team recommended first line palliative endocrine therapy (ET) with aromatase inhibitor (AI) - Anastrozole, which started at the end of April 2007. Supplementary treatment with vitamin D3 and calcium supplements were also initiated.

The patient was adherent to her treatment with no significant side effects besides mild joint pains, classified as grade 1, according to Common Terminology Criteria for Adverse Events (CTCAE) version (15). She was regularly followed-up with clinical examination, computed tomography (CT) scans of the thorax and abdomen as well as laboratory tests. Annual osteodensitometry showed mild progressive worsening of the osteopenia, without development of osteoporosis. Gradual regression of all tumor sites was noted after several months of treatment. Further on, there was a continuous sustained response of the primary tumor in the breast and in the axilla. After 13 months of ET in June 2008 the skin lesions were hardly visible and a

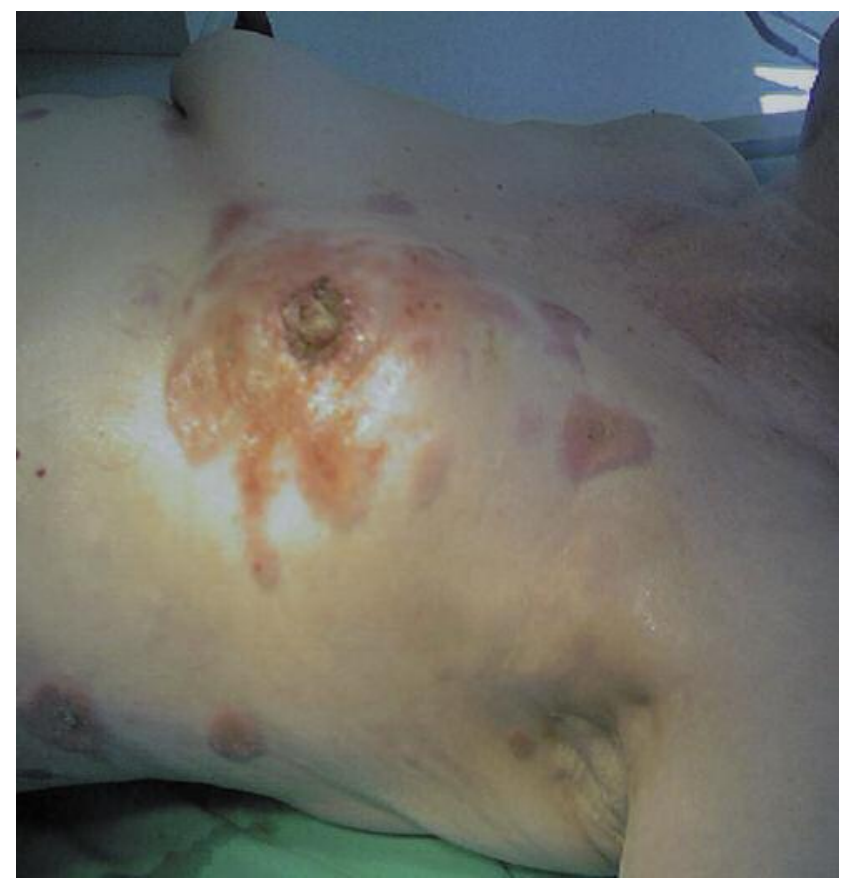

Figure 1. Left breast with a tumor mass with multiple nodular skin lesions.

partial clinical response was registered (Figure 4A and B). The primary tumor and all of the nodular skin lesions continued to gradually disappear and in February 2009 a complete response (CR) was achieved (Figure 5).

In May 2015, the treatment with Anastrozole was interrupted due to signs of osteoporosis (T-score - 2.6 despite optimal Vitamin D3/Calcium replacement). No additional treatment for osteoporosis (e.g. bisphosphonates) was available at that time in the absence of bone metastases. There were no clinical or imaging signs of progression of the oncological condition and the first line ET was shifted to Tamoxifen. The last clinical examination in December 2016 (after 113 months of ET) showed persistent CR in the breast, the lymph nodes and the SM.

No local procedures, such as surgery or radiotherapy were performed. The breast ultrasound, whole body CT-scans and the tumor markers were completely normal. The patient continued her ET for 116 months in total with no evidence of disease (NED) until December 2016 when she died at the age of 91 years from non-cancer related reason.

\section{Discussion}

Our case report shows a long-term survival and a sustained $\mathrm{CR}$ in a metastatic luminal lobular BC to first line ET of both the primary tumor and the only metastatic site - the cutaneous lesions. Metastatic Breast Cancer (MBC) is still 

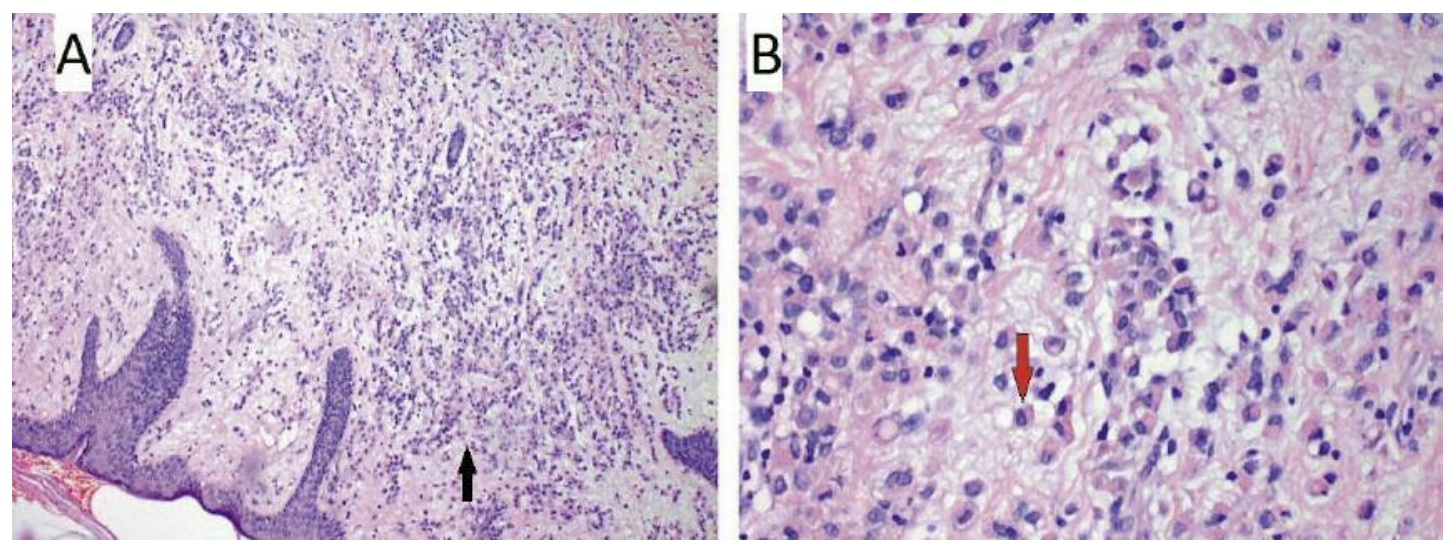

Figure 2. A) Classical lobular carcinoma with infiltration of the papillary derma (black arrow), HE stain $\times 10$. (B) Tumor cells with plasmacytic features, eosinophilic cytoplasm and cytoplasmic and nuclear nucleoli (red arrow), HE stain $\times 40$.
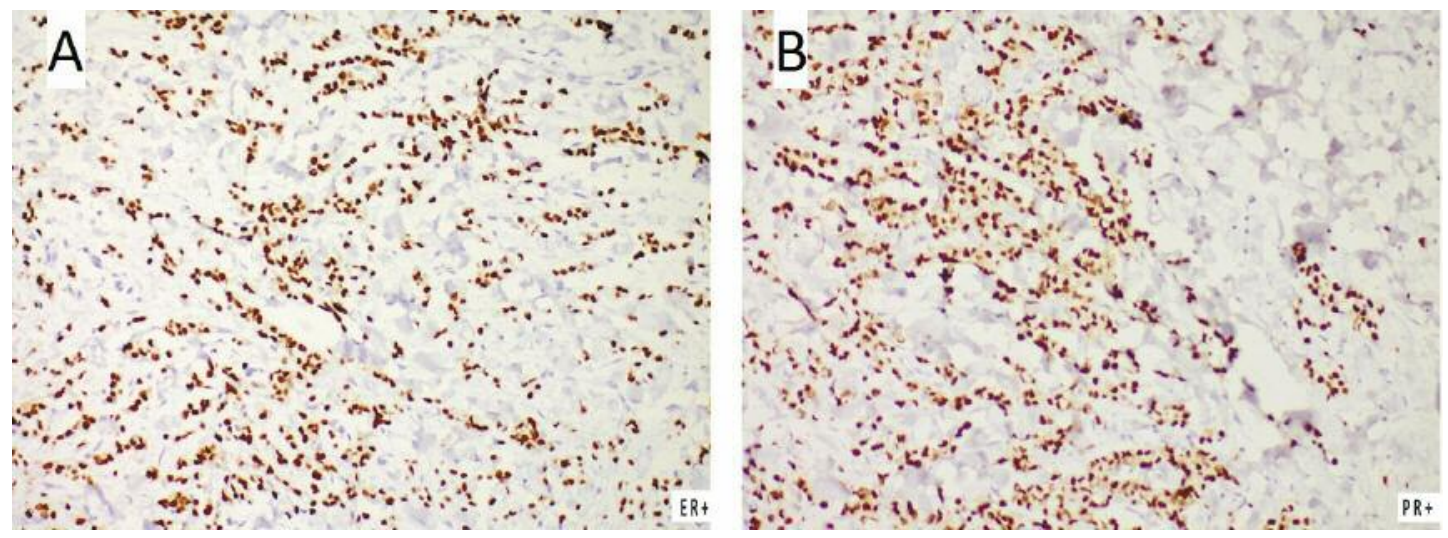

Figure 3. Typical immunohistochemistry with strong positivity for estrogen (ER;A) and progesterone (PR; B).
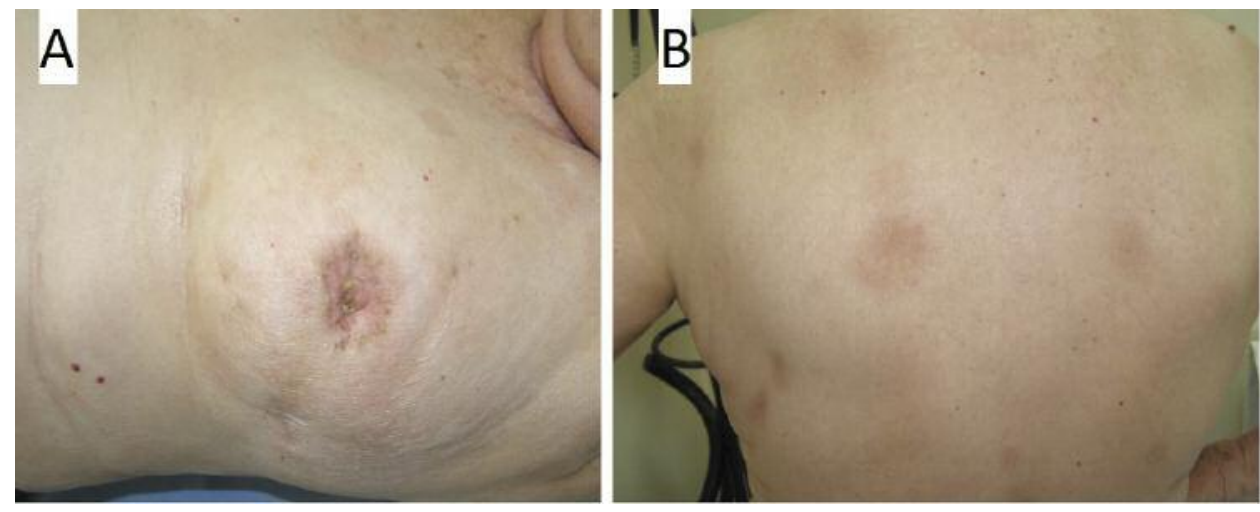

Figure 4. Partial clinical response following 13 months of treatment with Anastrozole (June 2008). A) The primary tumor in the left breast and skin lesions; B) Remaining post-healing hyperpigmentation of the skin metastases on the back. 

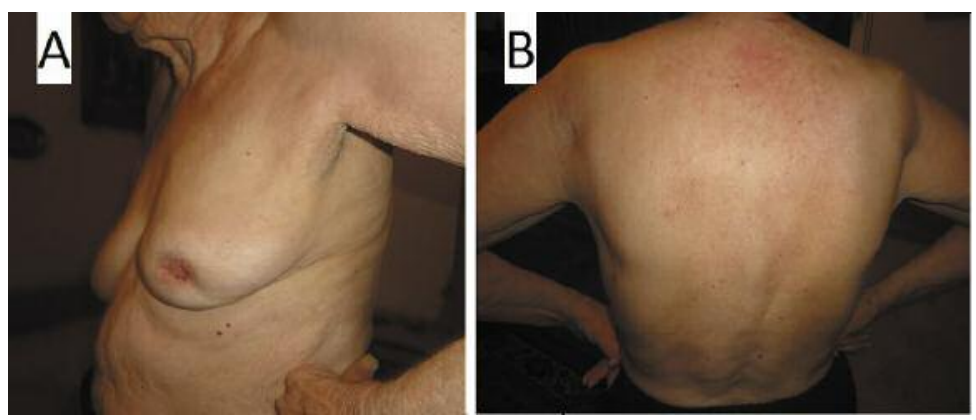

Figure 5. Complete clinical response following 21 months of treatment with Anastrozole (February 2009). A) No signs of a residual tumor in the left breast and total disappearance of all skin lesions. B) No evidence of the remaining skin metastases on the back.

considered an incurable disease, despite the fact that survival rates have improved in the last years (2). SM are relatively more frequent in $\mathrm{BC}$ and there are different mechanisms described and suspected for this skin tropism, such as direct invasion, extension through lymphatics, embolization through lymphatics and blood vessels, and accidental implantation during surgery (16).

Some of the patients may even be cured, however the reported cases more frequently refer to HER2 positive disease (1).

In general, skin metastasis is believed to be a manifestation of systemic metastasis, which carries a limited survival period, and thus presents itself as a terminal stage of malignancy. However, in the absence of internal visceral organ metastasis, some breast cancer patients with metastasis in the skin only may have a good prognosis and prolonged survival. Some of them may even be cured of the disease using targeted agents, such as trastuzumab, used in treatment of the HER2 positive subtype (17).

There are occasional reports of long-term control with CR of MBC, predominantly in low-burden metastatic disease (8). De novo metastatic skin lesions are relatively less frequent compared to the ones that present as a sign of metachronous disease progression. Management of SM may include, but is not limed to, available local techniques, such as radiotherapy and surgery, but in ER+ disease systemic treatment with ET, such as Tamoxifen or AI shows long-term results (17).

It is well known that lobular breast cancers tend to spread to "more unusual" secondary sites. The etiology of this is unknown, but it may refer to the loss of expression of the cellcell adhesion molecule E-cadherin in invasive lobular carcinoma (18). A retrospective analysis from 2016 has reported a median survival time (MST) of 55.5 months for all MBC subtypes (59.9 months for the luminal one) with clear improvement in the recent years (2000-2008) in comparison to 1977-1989 (19). Some cases of luminal MBC (7.3-11.6\%) have their first distant site of recurrence in the skin. The absence of E-cadherin also tends to be associated with the first distant metastases in the skin or the subcutaneous tissue (20).
In the adjuvant setting, Anastrozole has shown a better disease-free survival (DSF) compared to Tamoxifen in postmenopausal women with early BC (21). Superior survival of all third generation AIs compared to Tamoxifen in the first line setting of $\mathrm{MBC}$ has been reported in a large meta-analysis with fewer side effects from treatment with Anastrozole compared to Tamoxifen $(22,23)$. All these logically lead to the consideration of AIs as a first line ET (2).

A recent study with 570 patients with $\mathrm{MBC}$ has reported a rate of $16 \%$ of achievement of no evidence of disease (NED) status, assessed by imaging following systemic or local therapy and this was registered about 11 months after the start of the treatment of choice. More than half of all MBC patients (67\%) were de novo stage IV at diagnosis and $37 \%$ were ER+/HER2- (24). With a median follow-up of 100 months, the 3- and 5-year overall survival (OS) rates for the entire group were $44 \%$ and $24 \%$, respectively, compared to $96 \%$ and $78 \%$ for patients, attaining NED. The likelihood of achieving NED in the metastatic setting was higher if local treatment strategies had been used, in non-obese patients with de novo $\mathrm{MBC}, \mathrm{ER}+$ and with only one metastatic site. Of note is also the fact that about $1 / 3$ of all patients that achieved NED in the metastatic setting maintained this first remission. Following progression, about $1 / 3$ of these patients achieved NED as a second remission, which in our view is quite remarkable (24).

Here, we report a very rare case with cutaneous metastases from lobular BC with CR to Anastrozole therapy, in which skin involvement is the first and only sign of metastatic disease. The patient had no family history of BC and was diagnosed with luminal ER+/HER2- lobular BC. She was not amenable to local techniques, as the metastatic spread was significant. For this reason, she only received first line ET with Anastrozole that induced a long-term CR in the primary tumor as well as in the metastatic lesions. The shift to Tamoxifen in the last 2 years was not due to disease progression but due to side effects and osteoporosis, as a consequence of the ET combined with AI. It is not rare in the clinical practice to change the therapeutic approach from 
AI to Tamoxifen or vice versa in postmenopausal women due to side-effects. In the absence of progression, a switch between ET with different mechanisms of action is not assessed in clinical trials but may represent a successful therapeutic alternative.

We have found a similar case with BC cutaneous metastases and CR following therapy with Anastrozole alone or complex therapy, but it does not refer to a lobular cancer (25).

To our knowledge this is the only published case of lobular breast cancer with de novo metastatic manifestation as multiple skin lesions, which achieved CR following AI treatment with such impressive long-term OS (116 months), more than double compared to cited data. If well tolerated, maintenance treatment, especially ET, may be continued on a long-term scale or lifelong, as about $1 / 3$ of these patients would remain with NED. Alteration to ET with a different mechanism of action seems beneficial in cases of long-term response and when taking into consideration potential toxicities. Even following progression, a second CR or NED might be achieved in about $30 \%$ of the cases, which remains an important and significant percentage in latter lines of treatment, even in MBC.

\section{Conflicts of Interest}

The Authors have no conflicts of interest or funding to disclose.

\section{Authors' Contributions}

All Authors contributed equally to this work, generated and edited the figures and wrote the article.

\section{References}

1 Forouzanfar MH, Foreman KJ, Delossantos AM, Lozano R, Lopez AD, Murray CJL and Naghavi M: Breast and cervical cancer in 187 countries between 1980 and 2010: A systematic analysis. Lancet 378(9801): 1461-1484, 2011. PMID: 21924486. DOI: 10.1016/S0140-6736(11)61351-2

2 Cardoso F, Senkus E, Costa A, Papadopoulos E, Aapro M, André F, Harbeck N, Aguilar Lopez B, Barrios CH, Bergh J, Biganzoli L, Boers-Doets CB, Cardoso MJ, Carey LA, Cortés J, Curigliano G, Diéras V, El Saghir NS, Eniu A, Fallowfield L, Francis PA, Gelmon K, Johnston SRD, Kaufman B, Koppikar S, Krop IE, Mayer M, Nakigudde G, Offersen BV, Ohno S, Pagani O, Paluch-Shimon S, Penault-Llorca F, Prat A, Rugo HS, Sledge GW, Spence D, Thomssen C, Vorobiof DA, Xu B, Norton L and Winer EP: 4th ESO-ESMO International Consensus Guidelines for Advanced Breast Cancer (ABC 4). Ann Oncol 29(8): 16341657, 2018. PMID: 30032243. DOI: 10.1093/annonc/mdy 192

3 Lichter AS, Lippman ME, Danforth DN, D'Angelo T, Steinberg SM and DeMoss E: Mastectomy versus breast-conserving therapy in the treatment of stage I and II carcinoma of the breast: A randomized trial at the National Cancer Institute. J Clin Oncol 10(6): 976-983, 1992. PMID: 1588378. DOI: $10.1200 / \mathrm{JCO}$. 1992.10.6.976
4 Tevaarwerk AJ, Gray RJ, Schneider BP, Smith M Lou, Wagner LI and Fetting JH: Survival in patients with metastatic recurrent breast cancer after adjuvant chemotherapy: Little evidence of improvement over the past 30 years. Cancer 119(6): 1140-1148, 2013. PMID: 23065954. DOI: 10.1002/cncr.27819

5 Lookingbill DP, Spangler N and Helm KF: Cutaneous metastases in patients with metastatic carcinoma: A retrospective study of 4020 patients. J Am Acad Dermatol 29: 228-236, 1993. PMID: 8335743.

6 Lookingbill DP, Spangler N and Sexton FM: Skin involvement as the presenting sign of internal carcinoma: A retrospective study of 7316 cancer patients. J Am Acad Dermatol 22(1): 1926, 1990. PMID: 2298962.

7 Krathen RA, Orengo IF and Rosen T: Cutaneous metastasis: A meta-analysis of data. South Med J 96(2): 164-167, 2003. PMID: 12630642. DOI: 10.1097/01.SMJ.0000053676.73249.E5

8 Marcoval J, Moreno A and Peyrí J: Cutaneous infiltration by cancer. J Am Acad Dermatol 57(4): 577-580, 2007. PMID: 17368634. DOI: 10.1016/j.jaad.2007.01.034

9 Nanda R: "Targeting" triple-negative breast cancer: The lessons learned from BRCA1-associated breast cancers. Semin Oncol 38(2): 254-262, 2011. PMID: 21421115. DOI: 10.1053/ j.seminoncol.2011.01.007

10 Barinoff J, Harter P, Heitz F, Dittmer C, Kuemmel S, Hils R, Lorenz-Salehi F, Traut A and du Bois A: Survival rates in patients with primary metastatic breast cancer over a 10 -year period: A retrospective analysis based on individual patient data from a multicenter data bank. J Clin Oncol 31(15): 1577-1577, 2013. DOI: 10.1200/jco.2013.31.15_suppl.1577

11 Largillier R, Ferrero J-M, Doyen J, Barriere J, Namer M and Mari V: Prognostic factors in 1,038 women with metastatic breast cancer. Ann Oncol 19(12): 2012-2019, 2008. PMID: 18641006. DOI: $10.1093 /$ annonc/mdn424

12 Lobbezoo DJA, Van Kampen RJW, Voogd AC, Dercksen MW, Van Den Berkmortel F and Smilde TJ: Prognosis of metastatic breast cancer: Are there differences between patients with de novo and recurrent metastatic breast cancer? Br J Cancer 112(9): 1445-1451, 2015. PMID: 25880008. DOI: 10.1038/bjc.2015.127

13 Green FL, Page DL, Fleming ID, Fritzet AG, Balch CM, Haller DG and Morrow M: AJCC cancer staging manual, 6th edition. Ann Oncol 14: 345-346, 2003. DOI: 10.1093/annonc/mdg077

14 World Health Organisation: Assessment of fracture risk and its application to screening for postmenopausal osteoporosis. Report of a WHO Study Group. World Health Organ Tech Rep Ser 843: 1-129, 1994. PMID: 7941614.

15 Cancer Therapy Evaluation Program, Common Terminology Criteria for Adverse Events, Version 3.0, DCTD, NCI, NIH, DHHS, March 31, 2003. Available at: http://ctep.cancer.gov

16 Braverman IM: Skin manifestations of internal malignancy. Clin Geriatr Med 18(1): 1-19, 2002. PMID: 11913734.

17 Kong JH, Park YH, Kim JA, Kim JH, Yun J and Sun JM: Patterns of skin and soft tissue metastases from breast cancer according to subtypes: Relationship between EGFR overexpression and skin manifestations. Oncology 81(1): 55-62, 2011. PMID: 21934337. DOI: 10.1159/000331417

18 Sastre-Garau X, Jouve M, Asselain B, Vincent-Salomon A, Beuzeboc P and Dorval T: Infiltrating lobular carcinoma of the breast: Clinicopathologic analysis of 975 cases with reference to data on conservative therapy and metastatic patterns. Cancer 77(1): 113-120, 1996. PMID: 8630916. DOI: 10.1002/(SICI) 10970142(19960101)77:1\%3C113::AID-CNCR19\%3E3. 0.CO;2-8 
19 Kobayashi K, Ito Y, Matsuura M, Fukada I, Horii R and Takahashi S: Impact of immunohistological subtypes on the long-term prognosis of patients with metastatic breast cancer. Surg Today 46(7): 821-826, 2016. PMID: 26467559. DOI: 10.1007/s00595-015-1252-x

20 Sihto $\mathrm{H}$, Lundin $\mathrm{J}$, Lundin $\mathrm{M}$, Lehtimäki T, Ristimäki A and Holli K: Breast cancer biological subtypes and protein expression predict for the preferential distant metastasis sites: A nationwide cohort study. Breast Cancer Res 13(5): R87, 2011. PMID: 21914172. DOI: 10.1186/bcr2944

21 Howell A, Cuzick J, Baum M, Buzdar A, Dowsett M, Forbes JF, Hoctin-Boes G, Houghton J, Locker GY and Tobias JS: Results of the ATAC (Arimidex, Tamoxifen, Alone or in Combination) trial after completion of 5 years' adjuvant treatment for breast cancer. Lancet 365(9453): 60-62, 2005. PMID: 15639680. DOI: 10.1016/S0140-6736(04)17666-6

22 Mauri D, Pavlidis N, Polyzos NP and Ioannidis JPA: Survival with aromatase inhibitors and inactivators versus standard hormonal therapy in advanced breast cancer: Meta-analysis. J Natl Cancer Inst 98(18): 1285-1291, 2006. PMID: 16985247. DOI: $10.1093 /$ jnci/djj357
23 Nabholtz JM, Buzdar A, Pollak M, Harwin W, Burton G, Mangalik A, Steinberg M, Webster A and von Euler M: Anastrozole is superior to tamoxifen as first-line therapy for advanced breast cancer in postmenopausal women: Results of a North American multicenter randomized trial. J Clin Oncol 18(22): 3758-3567, 2000. PMID: 11078488. DOI: 10.1200/ JCO.2000.18.22.3758

24 Bishop AJ, Ensor J, Moulder SL, Shaitelman SF, Edson MA, Whitman GJ, Bishnoi S, Hoffman KE, Stauder MC, Valero V, Buchholz TA, Ueno NT, Babiera G and Woodward WA: Prognosis for patients with metastatic breast cancer who achieve a no-evidence-of-disease status after systemic or local therapy. Cancer 121(24): 4324-4332, 2015. PMID: 26348887. DOI: $10.1002 /$ cncr.29681

25 Santiago F, Saleiro S, Brites MM, Frutuoso C and Figueiredo A: A remarkable case of cutaneous metastatic breast carcinoma. Dermatol Online J 15(7): 10, 2009. PMID: 19903438.

Received May 20, 2019

Revised June 18, 2019

Accepted June 27, 2019 